\title{
Atlas escolares para la educación geográfica de niños y jóvenes
}

\author{
School atlases for the geographical education \\ of children and young people
}

\author{
José Jesús Reyes Nunez*
}

Recibido 10 de enero de 2019; aceptado 19 de febrero de 2020

\section{Resumen}

El objetivo de este artículo es presentar de manera concisa el surgimiento y desarrollo de los atlases escolares desde el siglo XVIII hasta nuestros días. Una vez definido su significado, se presentan brevemente las tareas que se realizan durante la edición de atlases de este tipo, así como algunos ejemplos. En esencia, las etapas de la creación de un atlas escolar no han variado mucho, sin embargo los soportes en que se publican, sí. Somos testigos de la evolución de los soportes: de las ediciones impresas en papel primero, después los soportes electrónicos (CD), y actualmente la web. Se ofrecen también ideas sobre las posibilidades que pueden brindar las aplicaciones hechas para dispositivos móviles y qué nuevas investigaciones se realizan para diversificar y optimizar el uso de los atlas escolares en el futuro.

Palabras clave: atlas escolar, cartografía escolar, web cartografia, tecnología móvil.

\begin{abstract}
The main aim of this article is to present concisely the beginning and development of school atlases from the 18th century to the present day. After defining the school atlases, those tasks that are faced during the edition of atlases of this type are briefly presented, as well as some examples. In essence, the stages of creating a school atlas
\end{abstract}

* Universidad Eötvös Loránd, Hungría, correo electrónico: jesusreyes@caesar.elte.hu. ORCID: http://orcid.org/0000-0001-6080-8667. 
have not changed markedly, changing only the medium in which they are published. We can witness how they developed from paper-printed editions first to electronic media (CD) and currently to the web. Ideas are also given on the options that applications made for mobile devices can offer and what new research is done to diversify and optimize the use of school atlases in the future.

Key words: school atlas, school cartography, web cartography, mobile technology.

\section{Introducción histórica}

Desde hace siglos se han usado mapas en la enseñanza, para complementar e ilustrar los conocimientos impartidos en asignaturas relacionadas con la Geografía, pero también en otras que precisan de la información espacial en su contenido, como por ejemplo tradicionalmente ha sido la Historia. Dos de los primeros atlases expresamente publicados para ser usados por alumnos son obra del cartográfo alemán Johann

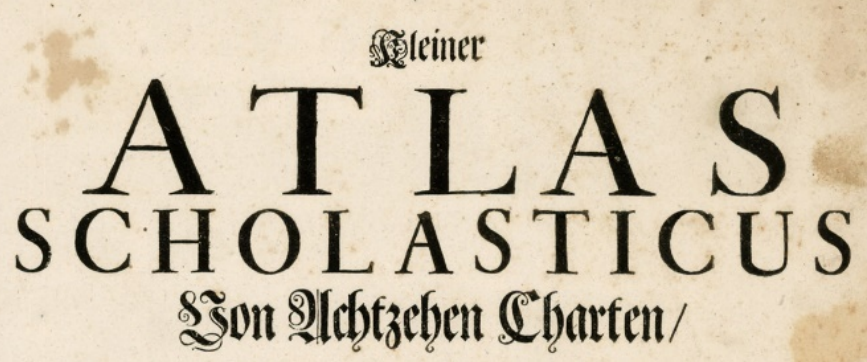

I. Bon bet $\Re$ Belt îugel.

I0. அon Şollant.

2. $3 o n$ Europa.

II. Bon det Sdweik.

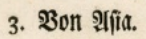

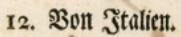

4. ßon शfrica.

13. ßon Deutíd)lant.

5. Bont Imerica.

I4. Bont Dainnemarcf.

6. Bon Spanien und Portugall.

15. Bon Sdweden und Rorwegent.

7. Bon frantreid).

I6. 3on ஒoblent.

8. Bon S̈roḱ: :Britantuien.

17. જon Lngarn unt Šried)enlant.

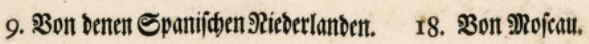

ediret

\section{von @obaum \$aptiffa \$omann/}

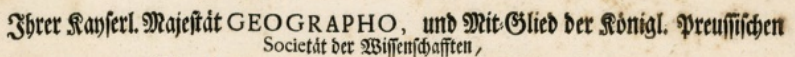

Figura 1. Portada del Kleiner Atlas Scholasticus de J. B. Homann con una lista de los mapas incluidos. Fuente:https://www.raremaps.com/gallery/detail/22948/title-page-kleiner-atlas-scholasticus $\underline{\text { homann-heirs }}$ 
Baptist Homann. El primero vió luz pública en 1710 bajo el título de Kleiner Atlas Scholasticus, contando con 26 mapas a color (Figura 1).

El segundo atlas fue publicado en Nurenberg en 1719 y su título en latín es Atlas Methodicus con el subtítulo "Explorandis Juvenum Profectibus in Studio Geographico...". En él se incluyeron 14 mapas para representar los países de Europa, Asia, África y América, también presenta cuatro representaciones del sistema solar según las ideas de Ptolomeo, Tycho Brahe, Copérnico y Descartes. Como detalle interesante, California aparecía dibujada como una isla en el mapa de América, y aparece también en la lista de nombres geográficos entre los nombres de las islas.

Durante el siglo XVIII se continuaron publicando atlases para las escuelas. En 1774, en Lyon (Francia), Jean-Marie Bruyset publicó su Atlas des enfans, ou nouvelle méthode pour apprendre la géographie..., con 24 mapas caracterizados por tener un contenido simplificado, que incluso fue traducido al inglés. A finales de ese mismo siglo, en 1792 sería publicado el primer atlas escolar en lengua española en la ciudad de Madrid, España. Su autor fue don Tomás López y se titulaba Atlas Elemental Moderno o colección de mapas para enseñar a los niños Geografía con una idea de la Esfera. El atlas, que contaba con un texto introductorio de 24 páginas y 27 mapas, se caracterizó por el grado de detalle de los mapas, que enriquecía el ejemplo del Atlas Metódico de Homann, incluyendo una introducción que ofrecía conocimientos de geografía astronómica y seguidamente dos páginas que estaban dedicadas a representaciones gráficas relacionadas con este tema (Figura 2).

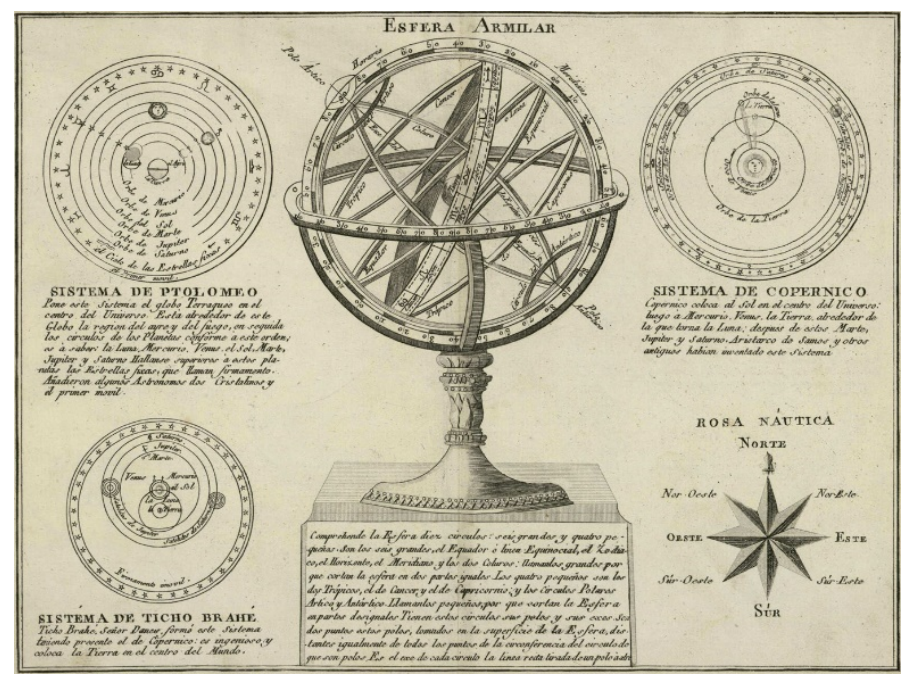

Figura 2. Una de las páginas ilustrando el texto de la introducción del Atlas Elemental Moderno de don Tomás López.

Fuente: http://bdh-rd.bne.es/viewer.vm?id=1110\&page=19 
En 1795 es publicado el primer atlas escolar en los Estados Unidos: The General Atlas for Carey's Edition of Guthrie's Geography Improved, aunque según Patton (1999) los mapas de este atlas ya habían sido publicados anteriormente por Mathew Carey en atlases dedicados a los adultos.

El siglo XIX constituyó un período de vital importancia en el desarrollo de lo que posteriormente llamaríamos cartografía, y en especial de la publicación de los atlases escolares, fue cuando el uso de estos atlases se extendió con mayor fuerza en muchos países. Como ejemplo cito solamente dos países ubicados en dos continentes distintos: entre los países del continente europeo selecciono a Francia, que se destacó por la variedad y profesionalidad de los mapas temáticos que se incluían en los libros de texto. El autor más reconocido internacionalmente fue el profesor, geográfo, economista e historiador Pierre Émile Levasseur (1828-1911), quien posteriormente fuera nombrado presidente honorario de la Sociedad Geográfica Francesa. Levasseur no se limitó a ilustrar y complementar los textos de sus libros con mapas temáticos, sino también creó atlases escolares que se caracterizaron porque en su introducción contenía una presentación gráfica y textual muy detallada de los conceptos elementales relacionados con la astronomía, los mapas y la geografia.

Otro país con un desarrollo muy diversificado de los atlases escolares fue Estados Unidos. Durante el siglo XIX se fundaron casas editoriales que se dedicaran a publicar este tipo de materiales, y nombres como Sidney E. Morse, Nathaniel G. Huntington, A. N. (Jessie) Olney y William C. Woodbridge, entre otros, se identificaran con los atlases escolares por ellos publicados. Mencionaría tres detalles interesantes, que corroboran el auge que tuvieron los atlases escolares estadounidenses en este siglo: después de su viaje por América del Sur y Central, Alejandro von Humboldt hizo las mediciones necesarias para crear el primer mapa del mundo de isotermas, que presentó la variación de la temperatura en la Tierra. Este mapa fue dibujado por primera vez en 1823 por William C. Woodbridge (Figura 3) y sería publicado en su atlas escolar de 1826 (School Atlas designed to accompany Woodbridge's Rudiments of Geography), que antecediéndose a su publicación en Europa, que ocurre años más tarde, con el famoso Atlas Berghaus de 1838. Esta obra de Woodbridge se considera un hito en la historia de la cartografía escolar estadounidense, ya que por primera vez incluía tres mapas temáticos: el ya mencionado de isotermas, otro de animales del mundo y uno de moral y política.

En los Estados Unidos también se publicó por primera vez un atlas escolar hecho por una mujer, que al mismo tiempo fue el primer atlas histórico escolar publicado en ese país: el Ancient Atlas de Emma Willard, quien era una maestra devenida la primera mujer cartógrafa estadounidense y que fue publicado en New York en 1828. Por último, mencionaría el atlas escolar publicado por Jesse Olney, cuyo título original en inglés es School Atlas to Accompany the Practical System of Geography for Schools and Families y que tuvo un total de 91 ediciones en un período de 21 años, 
entre 1829 y 1860 (Patton, 1999). Todo este desarrollo impetuoso propio del siglo XIX se hace mucho más interesante cuando leemos lo que el cartógrafo Erwin Raisz escribió en su subcapítulo dedicado a los atlases escolares en su célebre libro de texto Cartografía General en 1948: "Los atlases escolares no son comunes en los Estados Unidos, ya que los libros de texto de Geografía contienen todos los mapas necesarios" (Raisz, 1948: 218). Catorce años más tarde confirmaría esta afirmación en su libro Principios de Cartografía (Raisz, 1962: 102).

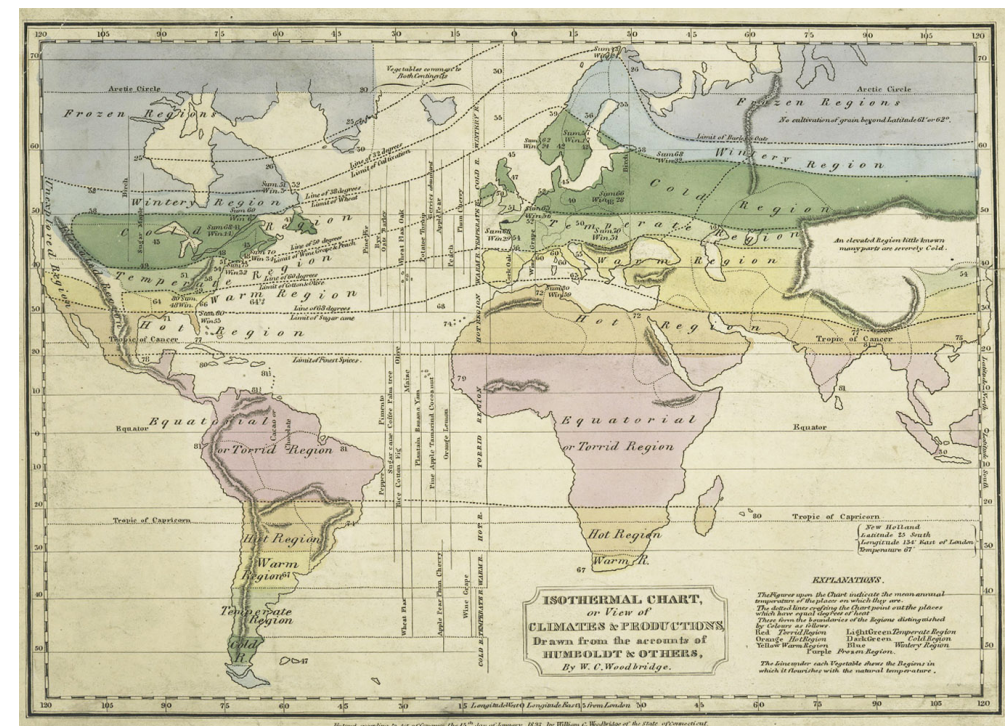

Figura 3. "Mapa del mundo de isotermas", realizado por William C. Woodbridge en 1823. Fuente: https://commons.wikimedia.org/wiki/File:Woodbridge isothermal_chart.jpg

El siglo XIX también trajo consigo la fundación y desarrollo de firmas cartográficas o casas editoriales especializadas en temas geográficos, cuya actividad se extendería también al siglo XX. Entre ellos tenemos, por ejemplo, el Atlas General VidalLablache: histoire et géographie, publicado por primera vez en 1894 en París, Francia, y que tendría nuevas ediciones en el siglo siguiente, o los atlas escolares publicados en Alemania y Austria por firmas como Gotha \& Justus Perthes (que publicó por primera vez en 1888 el Methodischer Schulatlas de Sydow y Wagner y cuya última edición fue la número 23 en 1944) o la editorial Hölzel que desde 1861 hasta el día de hoy continúa publicando su Kozenn-Atlas. 
Otras naciones más pequeñas también trataron y tratan de mantener la publicación de atlas escolares para sus escuelas. Como ejemplo característico podría citar a Hungría, cuyo primer atlas escolar fue publicado en el año 1800 en la ciudad de Debrecen, a unos $230 \mathrm{~km}$ de la actual Budapest. Su título fue Nuevo Atlas Escolar para alumnos de primaria y su autor el profesor Ézsaiás Budai. El atlas contenía solo 12 mapas y no incluía un mapa de Hungría: el país estaba representado solamente en un mapa regional. Durante los siguientes 90 años los atlases escolares húngaros eran traducciones de atlases alemanes o austríacos. En 1890 un conocido cartógrafo, Manó Kogutowicz, funda el Instituto Geográfico de Hungría que, a pesar de su nombre, era una compañía privada que se dedicaría principalmente a la publicación de atlases, mapas murales y esferas para las escuelas. Hasta la Primera Guerra Mundial, el Instituto publicó siete atlases escolares geográficos y tres atlases escolares históricos, que tuvieron numerosas ediciones, aparte de 37 mapas murales físicos, 24 mapas murales de provincias húngaras, 13 mapamundis murales históricos y 10 mapas murales históricos de Hungría, sin contar la producción de mapas ciegos para tareas escolares y globos terrestres para las aulas (Klinghammer, 2001). Su labor no fue solo reconocida en el país, sino también a nivel internacional, ganando medallas en exposiciones y congresos internacionales. Podemos afirmar que Kogutowicz y su instituto sentaron las bases del desarrollo de la cartografía escolar moderna en Hungría (Figura 4).

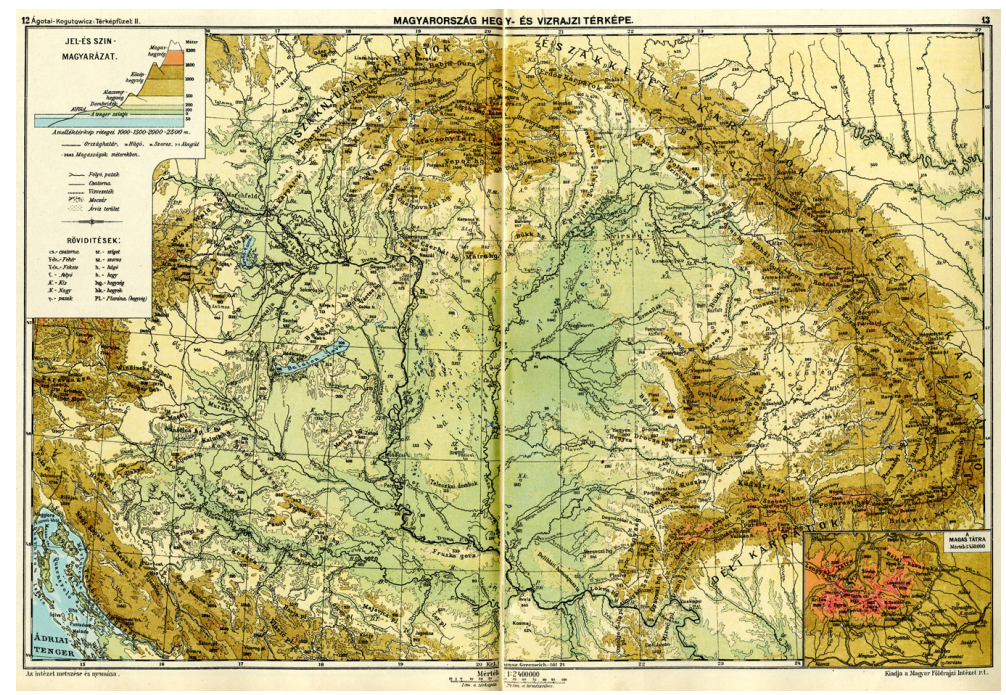

Figura 4. Versión publicada en 1906 del primer mapa físico del valle de las Cárpatos hecho con curvas de nivel en el Instituto Geográfico de Hungría. 


\section{Definiendo los atlases escolares}

En la cartografía podemos encontrar que la definición de atlases ha ido modificándose con el paso del tiempo. Citaría tan solo un ejemplo válido para los atlases tradicionales impresos: en 1948 el cartógrafo norteamericano de origen húngaro Erwin Raisz en su libro Cartografía General definió un atlas como una colección de mapas, generalmente en un solo volumen, publicado en un mismo idioma, con simbolización y proyecciones uniformes, pero no necesariamente con la misma escala (p. 216). En 1962, Raisz ofreció una definición muy similar, con la única diferencia de que ya no se limita a hablar de un atlas como una publicación en un solo volumen, sino en varios volúmenes o con hojas sueltas que permiten una actualización más rápida.

Con la llegada de la era digital a la cartografía, se consideró que la definición de atlas también debía ser adaptada a esa nueva tecnología, utilizandóse un estilo de redacción que puede ser aplicado tanto a los atlas impresos como a los electrónicos o digitales. En 1993, Olev Koop define un atlas como "una colección sistemática y coherente de datos geográficos, en formato análogo o digital, representando un área particular y/o uno o más fenómenos geográficos, basada en una narrativa junto con herramientas para la navegación, búsqueda de información, análisis y representación" (Koop, 1993).

Si visitamos la página web de la Comisión de Atlases de la Asociación Cartográfica Internacional (ACI, ICA por sus siglas en inglés) encontramos varias definiciones, entre ellas la que fue redactada por el profesor checo Vit Voženilek (2014): "la cartografía actual considera un atlas como una serie de mapas compilados con un objetivo, que han sido sistemáticamente organizados de acuerdo con el contenido temático, la extensión espacial y el punto de vista temporal, y ensamblados usando un lenguaje cartográfico común”.

Estas definiciones también son aplicables a los atlases escolares, aunque estos tienen sus características muy propias, por ejemplo su publicación se realiza casi siempre en un solo volumen. Una definición de atlas escolares podemos encontrarla en el libro electrónico The World of Maps, publicado por la ACI en su página web: "los atlas escolares son aquellos que introducen a los estudiantes en los aspectos físicos y socioeconómicos de la geografía mundial" (Ormeling, 2014). Erwin Raisz no define los atlas escolares en sus libros, pero en su opinión estos atlases deben cumplir tres funciones: ser fuente de información, dar imágenes claras y fáciles de recordar de los patrones geográficos, así como estimular el interés de los alumnos (Raisz, 1948). Se puede afirmar que la diferencia entre un atlas escolar y otros tipos está determinada fundamentalmente por la edad y conocimientos del "público" o los "usuarios" al que está dirigido, o sea de los alumnos, lo que significa que en el momento de crear los mapas se debe considerar cuáles métodos de representación utilizar, así como cuál es el contenido que los alumnos estudian en dependencia del nivel de enseñanza que cursan. 


\section{¿Qué principios deben regir la publicación de un atlas escolar?}

Al ser el atlas escolar una obra para ser usada por alumnos, su redacción debe cumplir requisitos específicos. Uno de ellos es la selección del lenguaje gráfico usado para la representación de datos. Patrick Wiegand en su libro Learning and teaching with maps enfatiza acertadamente que los métodos usados para la representación de datos debe adecuarse a las edades a la que se dirige el atlas, pero al mismo tiempo precisa ser variado (Wiegand, 2006). Eso significa que deben utilizarse (y deben adaptarse) métodos de diferentes tipos (símbolos proporcionales, coropletas, diagramas, etc.), para que los alumnos vayan adquiriendo experiencia en la lectura e interpretación de los datos representados en los mapas. Otro requisito tan importante como el anterior es que su contenido debe incluir los conocimientos que se orientan en los currículos nacionales, para que el atlas pueda ser considerado un verdadero complemento de los libros de texto que utilizan los alumnos, siendo atlas creados para complementar o enriquecer aquellos conocimientos que son ubicados espacialmente (o, para usar una denominación más moderna, que son georeferenciados) y que se imparten en las escuelas de nivel primario y secundario. De esta manera se puede inferir que es muy importante que en cada país se pueda producir sus propios atlases escolares, aunque por diferentes razones existen aún países en los que no se publican y por ello en las escuelas se hace uso de aquellos publicados por firmas o editoriales cartográficas de otros países, pero que son internacionalmente reconocidas por la calidad de sus trabajos. Esto es posible porque existen conocimientos básicos generales que se pueden considerar comunes en todos los sistemas educativos y que usualmente son incluidos en los atlases escolares de cualquier país, por ejemplo los mapas físicos y políticos de los continentes. Pero si analizamos al detalle su contenido, podremos constatar que hay, por lo menos un tema de los currículos nacionales que no pueden presentar en absoluto o de manera totalmente satisfactoria: la información geográfica, económica, social, cultural, etc. más detallada que se relaciona con el país en cuestión y sus regiones, provincias, etc. También podemos encontrar excepciones: cuando una firma cartográfica de un país publica un atlas escolar, cuya venta está específicamente dirigida otro país, por ejemplo la serie de atlases escolares que la editorial Oxford University Press publica periódicamente para ser vendido en la India: en las 160 páginas de la tercera edición del Oxford Student Atlas for India encontramos 32 mapas dedicados al subcontinente indio, además de ofrecer como anexo un mapa de la India (Oxford University Press, 2019).

La redacción de los mapas para los atlases escolares es un proceso muy delicado, que pone a prueba la experiencia del cartógrafo: hay que crear mapas que pueden parecer simplificados si los comparamos con los de un atlas mundial hecho para el público en general, pero ese proceso de selección del contenido y su generalización cartográfica siempre debe realizarse tomando en cuenta las indicaciones que ofrecen los currículos nacionales, sincronizando el contenido de los mapas con el de los libros 
de texto. No es posible incluir una descripción, ni tan siquiera abreviada, de cada una de las fases del proceso de redacción de los mapas. En su lugar, analicemos brevemente una de las tareas que constituye un momento clave en la edición del atlas: la selección de los nombres geográficos que aparecerán en los mapas y la toma de decisión en cómo escribir correctamente esos nombres. Este es un proceso también complejo, en el cual los cartográfos cuentan con la colaboración de especialistas en Letras. Dentro de esta tarea, un ejemplo específico es el uso de exónimos. Los exónimos son aquellos nombres empleados en un idioma determinado para aquellos objetos geográficos situados fuera del área, donde ese idioma es oficial y que se diferencian del nombre original. Por ejemplo: en el idioma español Nueva York y Londres son los exónimos de New York y London. La práctica usual en los atlases escolares es tratar de utilizar exónimos siempre que es posible y en algunos países incluso, junto con el exónimo se coloca el nombre en el idioma original entre paréntesis, para ofrecer una mayor información al estudiante principalmente de nivel secundario. Al mismo tiempo, las Naciones Unidas recomiendan disminuir el uso de los exónimos (Tichelaar y Ormeling, 2009), aunque en la práctica cada país mantiene la tendencia a usarlos. En algunos países se hacen listas oficiales de exónimos que se pueden utilizar en los mapas, pero ello no es una práctica usual en todos los países.

La estructura de los atlases publicados para su uso en las escuelas tiene en general características comunes: la mayoría de estos atlases comienzan con una presentación gráfica de aquellos conocimientos básicos que se relacionan con la geografía y los mapas. El contenido a mostrar es muy variado y depende de los grados para los que se publica el atlas. Estas introducciones gráficas generalmente comienzan con conocimientos de carácter astronómico, uno de ellos puede ser la ubicación de la Tierra en el Sistema Solar y el movimiento de la Tierra alrededor del Sol y sobre su propio eje. Hay atlases en los que también se muestran gráficamente otros conceptos relacionados con las ciencias de la tierra: estructura interna del planeta Tierra, estructura y composición de la átmosfera, etc. Esto puede ser seguido por conocimientos elementales de cartografía matemática: la presentación de algunas proyecciones cartográficas y nociones relacionadas con el sistema de coordenadas geográficas. A continuación se puede incluir los métodos de representación del relieve, partiendo desde una imagen satelital hasta los principios básicos de las curvas de nivel. Más tarde se hace la introducción al concepto de escala en los mapas, presentando un territorio a diferentes escalas. Y finalmente se puede utilizar esta sección del atlas para presentar detalladamente la leyenda o las leyendas de sus mapas.

El orden de presentación de los mapas también puede variar en los atlases escolares. Se puede afirmar que la secuencia de mapas en un atlas escolar sigue un orden lógico basado en tres aspectos esenciales: la orientación en tiempo y espacio, la geografía de las geoesferas (litosfera, hidrosfera, atmósfera, clima y zonas geográficas) 
y la geografía de las sociedades (población, asentamiento y economía) (Kocsis, Márton y Jordan, 2019). El profesor brasileño Marcello Martinelli (2008) propone el siguiente orden para un atlas publicado según un currículo nacional: presentación de nociones básicas, colección de mapas partiendo del espacio más próximo al alumno a escala relativamente grande hasta los mapas físico y político del país, continuando con los mapas por continentes, para concluir con los mapas físico, político y temáticos del mundo, y otro de los husos horarios. Por ejemplo, los atlases escolares publicados en Hungría siguen un orden muy similar a este, pero el Atlas Geográfico Escolar Rumano (Mândruț, 2018) sigue un orden inverso de los mapas, partiendo desde el mundo para finalizar con los mapas político y físico del país, al igual que algunos de los atlases escolares editados en Bulgaria en los últimos 15 años (Bandrova, 2006).

Los atlases escolares tampoco se limitan a ser complemento de los libros de texto relacionados con la Geografía. Un ejemplo típico de ello son los atlases escolares históricos, que se publican para las clases de Historia a diferentes niveles en muchos países. Pero hay también ejemplos que pueden ser considerados curiosos por su singularidad e incluso rareza: en Hungría se publica un Atlas Escolar de Literatura para los grados 5 al 8 de las escuelas primarias (56 pp.) y otro para las escuelas secundarias (72 pp.), que contienen la información mapificada de los conocimientos que se imparten en la asignatura de Literatura, desde la vida de los escritores y poetas hasta los lugares donde se desarrolla la trama de los libros que se estudian, además de incluir también mapas sobre la historia de la literatura.

Un caso excepcional y único en la publicación de atlases escolares puede considerarse la experiencia que se está desarrollando en Brasil, a partir de la década de los noventa. Considerando la extensión territorial de país y la diversidad de sus regiones, los educadores brasileños decidieron comenzar la publicación de los llamados atlases escolares municipales, que como su nombre lo indica, contiene información textual y gráfica muy detallada sobre un municipio del país. El primer atlas escolar municipal se publicó en 1994 en Ijuí (Rio Grande do Sul) con la coordinación de la profesora Helena Callai (Lastoria, 2007) y los últimos atlases se han publicado en Pirenópolis (2018) y Distrito Federal (2019). No se tratan de atlases escolares tradicionales formados fundamentalmente por mapas. Tal vez sería más correcto caracterizarlos como un libro de texto profusamente ilustrado y acompañado también de numerosos mapas. El atlas en ocasiones también incluye ejercicios para ser contestados por los alumnos, o para investigar por cuenta propia, su formato es variado, así como la impresión, que puede ser a color o en blanco y negro. Su característica más meritoria es que ofrece conocimientos propios de la región donde vive el alumno, así como la masividad de la colección, ya que se han publicado numerosos atlases de este tipo en los 25 años transcurridos desde sus inicios. 


\section{Atlases escolares para la educación del futuro}

\section{Atlases escolares electrónicos}

Los atlases escolares tampoco podían ser una excepción del impacto ocasionado por la revolución digital que la aparición de las computadoras personales generó a partir de la década de los ochenta. En los años noventa, se comenzaron a desarrollar investigaciones y publicarse los llamados atlases electrónicos (también conocidos como digitales). Las primeras versiones digitales de atlases (incluyendo también los escolares), en muchos casos se publicaban en un disco compacto que acompañaba la versión impresa, utilizando las nuevas herramientas que ofrecían las técnicas digitales. Estos son aquellos atlases que en 1996 Kraak y Ormeling identificaban como "viewonly atlases" (atlases solo para visualización o lectura) y que definían como una extensión de los atlases impresos, brindando nuevas opciones propias del mundo digital (acceso rápido y "al azar" de los mapas a través de un menú, opción de visualizar en pantalla una parte aumentada del mapa, etc.) pero usando solamente mapas estáticos. Otro tipo de atlases electrónicos o digitales son los llamados interactivos, que permitían que el usuario decidiera cómo deseaba representar los datos a los que tenía acceso, por ejemplo cambiando los colores con que se representaban los datos o clasificando esos datos de diferentes maneras. Por último, también se desarrollaron los atlases analíticos, que como su nombre indica, permitían operaciones más complejas con los datos a través de los mapas o combinando los diferentes datos para crear mapas nuevos (Kraak y Ormeling, 1996).

Los discos compactos (CD) que acompañaban a los atlases escolares generalmente incluían materiales multimedia como vídeos de temas cartográficos (historia de los mapas, proyecciones, tipos de mapas, etc.), animaciones para mostrar diferentes conceptos geográficos o astronómicos (movimiento de rotación y traslación de la Tierra), mapas en formato raster (imagen) para su uso en las clases o por los alumnos en sus tareas individuales, juegos interactivos educativos y tablas de datos geográficos, económicos, etc. De esta manera, los discos compactos convertían al atlas escolar en una material educativo más íntegro, que brindaba múltiples opciones novedosas al alumno (y también al maestro) para enriquecer sus conocimientos. El uso de discos compactos para complementar el atlas se mantuvo hasta los primeros años de la década del 2010, cuando ya estaba ganando popularidad entre las generaciones más jóvenes el uso de los dispositivos móviles y en especial de los telefónos móviles inteligentes (smartphones). Adaptándose a las más novedosas tecnologías, los atlases escolares también sustituyeron los discos compactos por una opción más sencilla que combina el uso de los dispositivos móviles con Internet: los materiales multimedia se almacenan en un servidor con acceso en la web, y se crearon aplicaciones que permitían conectar el atlas con el dispositivo móvil. Esta solución se expande sin mayores obstáculos, no solo por su facilidad para usarla, sino porque 
también significa un ahorro material y financiero para las firmas cartográficas. Así, en la actualidad se hace usual encontrar en una esquina de la portada de un atlas escolar un texto breve pidiendo que se descargue una aplicación o presentando también un código QR para tener acceso a más mapas, ejercicios interactivos, animaciones, videos, juegos, etc.

\section{Atlases escolares y tecnologías móviles}

La opción de usar aplicaciones con un dispositivo móvil para acceder a materiales anexos a un atlas escolar puede ser considerada tan solo una especie de introducción a la verdadera adaptación de estos atlases al llamado mundo móvil. Para que eso ocurra, debemos ofrecer una respuesta cartográfica y tecnológicamente bien fundamentada a la pregunta: ¿cómo pueden servirse los atlases escolares de Las nuevas soluciones que se desarrollan para la cartografía móvil (por ejemplo los Locationbased services, LBS)?

Actualmente, en diferentes tiendas virtuales, se pueden comprar atlases supuestamente creados para los dispositivos móviles. Si los analizamos, veremos que en muchas ocasiones se trata de "rediseños" y solo en contados casos realmente "reeditados" para los dispositivos móviles. La mayoría está compuesto solamente por mapas estáticos (a menudo versiones en formato raster de los mapas de un atlas), haciendo un uso mínimo de las nuevas opciones brindadas por esos dispositivos, limitándose a diseñar un entorno adaptado al tamaño pequeño de la pantalla y a aprovechar la ventaja que significa el uso de la pantalla táctil para la selección de opciones o simplemente "hojear el atlas".

Pero un atlas escolar solo puede ser llamado "móvil" si es capaz de aprovechar las ventajas que ofrece esta tecnología y que no pueden ofrecer los atlases publicados en otros soportes electrónicos, por ejemplo en discos compactos. Reichenbacher (2001) definió claramente lo que debe ser la cartografía móvil: "es aquella que trata de las teorías y tecnologías de la visualización cartográfica dinámica de los datos espaciales y su uso interactivo en dispositivos portátiles en cualquier momento y en cualquier lugar bajo la consideración especial del contexto actual y de las características de los usuarios".

Si nos basamos en esta definición, entonces se puede afirmar que la edición y diseño de un atlas escolar para dispositivos móviles debe satisfacer tres condiciones concretas (Reyes, 2013):

1. Posicionamiento en espacio y tiempo (positioning in space and time): la primera condición que posibilita la ejecución de las dos siguientes. Este posicionamiento es totalmente automático, cuando el dispositivo móvil dotado de GPS determina la situación geográfica de la persona que lo usa así como la fecha y la hora (a tiempo real) de la tarea que está solucionando en ese momento. 
2. Reconocimiento del contexto (context awareness): después del posicionamiento, el atlas escolar puede identificar y coleccionar las informaciones relacionadas con el medio ambiente (contexto) en que se encuentra el usuario (el alumno). Estos datos pueden ser colectados por los llamados "geomotores" (geo-engines), que fueron definidos por Reichenbacher (2001). Los datos pueden ser utilizados conjuntamente con aquellos datos más generales que son almacenados previamente en las bases de datos que utiliza el atlas, por ejemplo, datos meteorológicos de una región determinada que han sido recogidos durante años u otras informaciones relacionadas con el contexto actual y que pueden ser culturales, históricas, sociales, etc.

3. Representación adaptativa (adaptive representation): solución gráfica para representar los datos seleccionados durante el reconocimiento del contexto. Estos deben ser representados en un mapa dinámico, cuyo contenido temático puede ser automáticamente modificado según la variación de los datos a tiempo real. Esto es lo que Safiza (2007) llama un modelo cartográfico flexible (flexible cartographic model) característico de las aplicaciones de tipo LBS.

\section{¿Hasta qué punto precisamos usar de un modelo cartográfico flexible en un atlas escolar?}

El uso de un modelo cartográfico flexible no tiene que ser de carácter obligatorio para todos los mapas de un atlas escolar móvil, porque hay escalas y temas que tradicionalmente aparecen en los atlases escolares y no necesitan de una actualización a tiempo y espacio real. Un ejemplo de este tipo de mapas puede ser un mapa físico del mundo u otro que presente el relieve e hidrografía de un continente, una región o de un país.

Los modelos cartográficos flexibles pueden ser utilizados para complementar y actualizar la información representada en algunos mapas temáticos a mayor escala después del reconocimiento del contexto del alumno. Incluso podemos permitirnos ir mucho más allá de los límites y objetivos tradicionales de los atlases escolares al aplicar las soluciones que brinda la tecnología móvil: el uso de los geomotores definidos por Reichenbacher (2011) nos ofrece la base tecnológica necesaria para expandir, multiplicar las funciones del atlas escolar y convertirlo en un material educativo interactivo mucho más completo. Este material lúdico no incluiría solo mapas, sino también ejercicios y juegos interactivos que serían automaticamente adaptados al contexto del alumno a través de su dispositivo móvil. Podríamos llamarlos geoejercicios adaptivos (adaptive geo-exercises), que combinarían las informaciones obtenidas a tiempo y espacio real con aquellas que fueron previamente almacenadas en la base de datos del atlas. 
Para ejemplificar su uso podríamos imaginar una clase de Geografía en una escuela secundaria, en la que los alumnos estudian una lección sobre el clima. Si combinamos los datos climáticos previamente almacenados en nuestro atlas escolar (que pueden ser datos de temperatura, precipitaciones o presión atmosférica en diferentes ciudades del mundo) con los datos obtenidos a tiempo real sobre el estado del tiempo en la ciudad donde vive el alumno, entonces los ejercicios se le plantearán usando ambos tipos de datos, cobrando mayor actualidad y ayudándolo a una mejor comprensión de la enseñanza. Esta realmente no es una propuesta "futurística", porque el desarrollo de la tecnología móvil no solo nos brinda estas opciones en el presente, sino incluso son actualmente utilizadas en aplicaciones didácticas creadas específicamente para niños y jóvenes.

Es lamentable que las grandes firmas cartográficas actualmente no se muestren interesadas en desarrollar los atlases escolares en esta dirección. En años anteriores se experimentaron algunas soluciones, por ejemplo una firma muy reconocida internacionalmente comenzó a comercializar su Atlas Mundial como una aplicación para dispositivos móviles, que conjuntamente con las soluciones tradicionales permitía también, por ejemplo, obtener datos a tiempo real del estado del tiempo en un país seleccionado por el usuario. Desdichadamente solo desarrollaron esta aplicación durante algunos años, y la última versión de este fue publicada en el 2015.

\section{Atlases escolares y los servicios de mapas en la web}

Una posible alternativa a la falta de atlases escolares móviles puede ser el uso de los servicios de mapas en la web para representar el contenido que se enseña en las clases. De cierta manera podemos afirmar que la aparición en el mercado de Google Maps y Google Earth en el año 2005 significó "un antes y después" en la historia de la cartografía contemporánea. Los dos servicios de la firma Google revolucionaron el uso tradicional de los mapas por los usuarios: los mapas de ciudades y turísticos en el sentido más clásico de la palabra, quedaron prácticamente sustituidos por estas dos aplicaciones en un período de tiempo relativamente corto. Si hasta ese momento la cartografía digital y la tecnología SIG era un mundo limitado a los especialistas y a algunos pocos interesados, principalmente Google Maps pero también Google Earth convirtieron los mapas digitales, las imágenes satelitales y las herramientas de búsqueda más sencillas en un fenómeno de masas: es la primera vez en la historia de la cartografía que se pone en manos de los usuarios (es decir, en manos de un público con un conocimiento sobre el uso de los mapas que puede ser considerado promedio o por debajo del promedio) un producto prácticamente gratis y de funcionamiento fácil de aprender para satisfacer casi todas sus necesidades relacionadas con la orientación espacial.

El uso cada vez más masivo de las aplicaciones desarrolladas por la firma Google y otras firmas que surgirían después del 2005 (OpenStreetMap en el 2006, Bing Maps 
en el 2009 así como Apple Maps y HereWeGo en el 2012) traerían consigo el surgimiento de nuevos conceptos o la redefinición de otros en la cartografía de la web: información geográfica voluntaria (volunteered geographic information, VGI), contenido generado por el usuario (user generated content), geografía de masas (mass geography), cartográfos laicos (lay cartographers), datos a grande escala o masivos (big data) entre otros.

Su influencia no podía dejar de sentirse también en la educación. Solo los temores de los maestros de mayor edad al uso de las nuevas tecnologías o aquellos con una formación informática insuficiente, así como las limitaciones materiales de una escuela podían obstaculizar la expansión de su uso en las clases de Geografía u otras asignaturas. Profesores de educación superior e investigadores en numerosos países han estudiado y aún hoy continúan estudiando las opciones que brinda su uso en las escuelas. Algunos ejemplos:

- Proyecto "La conciencia global en la escuela - Comprender el mundo con mapas" (Global Awareness in School - Understanding the World with Maps) ejecutado por el Departamento de Geografía de la Universidad de Viena (Austria) y cuyo objetivo principal fue experimentar el estudio del uso de Google Earth para representar datos temáticos en mapas creados para las escuelas (Kriz, 2008).

- Proyecto "Museo de Globos Virtuales", desarrollado por el Departamento de Cartografía y Geoinformática de la Universidad Eötvös Loránd en Budapest, Hungría (Figura 5). Iniciado en el año 2007, actualmente este museo contiene los modelos virtuales de 152 globos terráqueos, estelares, lunares y de otros planetas. El más antiguo de ellos data de 1507. El objetivo de este proyecto es permitir el uso interactivo (rotación, ampliación, etc.) de los globos digitalizados y visualizados en Google Earth (Gede et al., 2013). La página web donde se puede acceder la versión en inglés del museo es: http://terkeptar.elte.hu/vgm/?lang=en

- Proyecto "Geotecnologías y recursos de multimedia en la enseñanza de la cartografía: percepción socioambiental del Rio Alcântara en el municipio São Gonçalo (Rio de Janeiro)". Investigación realizada para una tesis de maestría en la Universidad Estatal de Rio de Janeiro, cuyo objetivo fue organizar una encuesta con la participación de alumnos de una escuela del barrio de São Gonçalo en la ciudad de Niterói, para preguntar a los vecinos sobre temas relacionados con el medio ambiente al mismo tiempo que recolectaban informaciones sobre aquellos lugares en el barrio de interés histórico, social, turístico, etc. En la siguiente etapa del proyecto se enseñó a los alumnos cómo representar en mapas estas informaciones usando Google Earth (Barros de Sousa, 2014).

Estos proyectos nos permiten vislumbrar las posibilidades que brindan los servicios de mapas en la web para la aparición de los futuros atlases escolares que utilicen como base cartográfica estas aplicaciones. Mi opinión es que tanto estos servicios de 


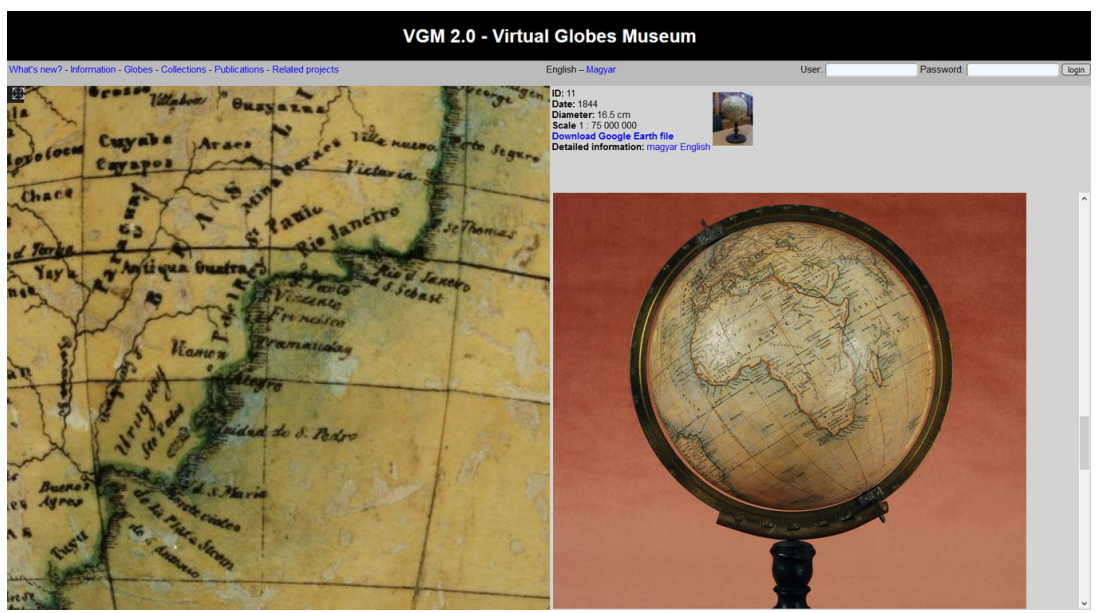

Figura 5. Uno de los 152 globos que contiene el Museo de Globos Virtuales.

Fuente: http://terkeptar.elte.hu/vgm/?lang=en

mapas (y principalmente Google Maps por su gran popularidad entre los usuarios laicos) pueden ser utilizados en la cartografía escolar, pero no deben (y aún no pueden) sustituir totalmente los mapas de un atlas escolar editado especialmente para determinados grados de la enseñanza primaria o secundaria. Esta afirmación está basada en que la cartografía temática prescribe que el contenido de las bases cartográficas se determine en dependencia del tema que se representa en un mapa y esta es una opción que los productos Google aún no ofertan, ya que solamente ofrece ocho tipos de bases cartográficas que se diferencian entre sí por el diseño, no permitiendo al usuario decidir cuáles capas (layers) son usadas o no en nuestro mapa.

El uso de los servicios de mapas también puede ser útil en otras tareas inherentes a los atlases escolares, ya en el siglo XIX era tradición incluir una introducción para representar y explicar gráficamente diferentes conceptos relacionados con los mapas. Un ejemplo típico es la descripción gráfica sobre cómo varía la representación de un territorio desde las grandes escalas a las más pequeñas. Este es un tema que Google Maps o Google Earth puede asumir por completo y de forma mucho más efectiva e interactiva que la cartografía tradicional. En un atlas escolar tradicional se presenta este proceso a través de un territorio muy conocido dentro del país o tomando como punto de partida una escuela "imaginaria", para ir aumentando a través de diferentes fragmentos de mapas el territorio que se muestra y así ir disminuyendo también la escala de los mapas que se utilizan en esta representación. Los productos Google pueden cumplir esta misma tarea automáticamente, ya que después que el alumno 
identifica su ubicación en el mapa (por ejemplo, dónde está su escuela) puede disminuir interactivamente la escala para mostrar cómo varía la representación a medida que aumenta el territorio a visualizar en la pantalla (Figura 6).

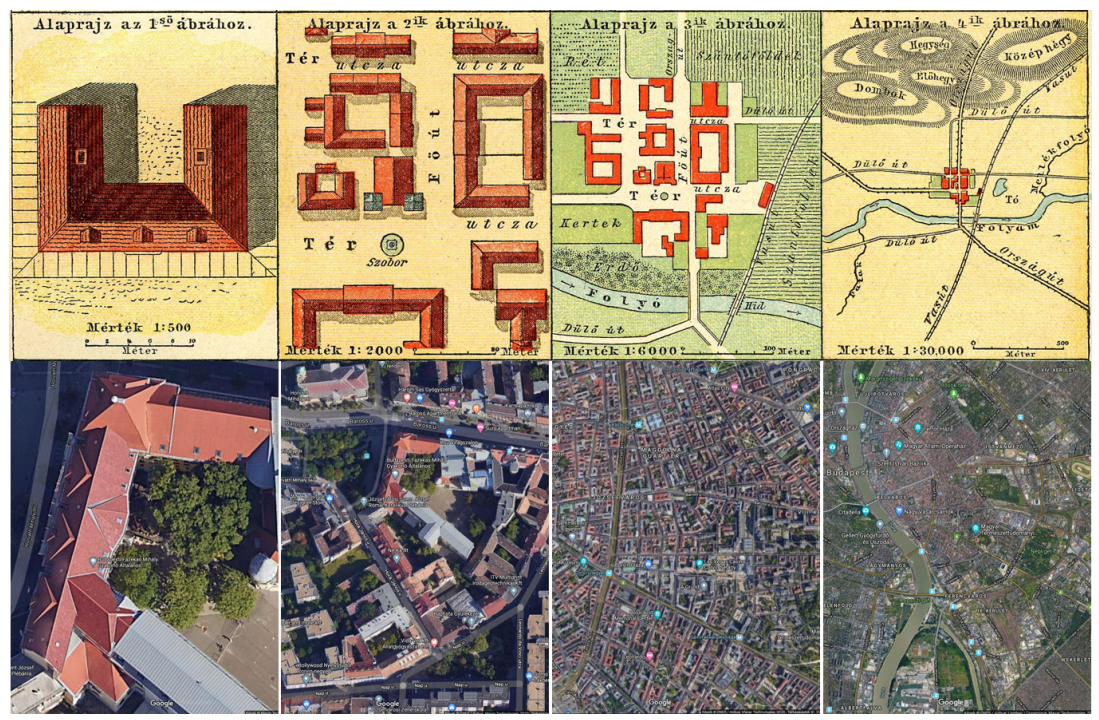

Figura 6. La parte superior muestra cómo se ilustraba la variación de la escala en un atlas escolar húngaro de $1890 \mathrm{y}$ en la parte inferior vemos el mismo proceso en Google Earth.

El uso de los servicios de mapas en la cartografía escolar (y fundamentalmente de Google Maps o Apple Maps) es ventajoso también por otros motivos más prácticos: a partir del año 2005 el diseño de estas aplicaciones se ha hecho familiar para millones de usuarios en todo el mundo (incluyendo las generaciones más jóvenes que aún estudian en las escuelas), además de ser una plataforma empleada en numerosas aplicaciones cartográficas desarrolladas para dispositivos móviles. Todo ello contribuye a hacer más rápido y sencillo el aprendizaje del uso de un atlas escolar por los usuarios. Esta ventaja fue aprovechada por Reyes y Kiss (2018) en un proyecto que permitió ver cómo estudiantes de noveno grado de una escuela secundaria húngara podían utilizar Google Maps y la aplicación Google My Maps para hacer ellos mismos sus mapas, después de haber estudiado en clases la unidad dedicada a la Geografía del agua. Se dedicó un turno de clases (45 minutos) para enseñar el uso de esa aplicación, al final de la cual los alumnos se organizaron en parejas y como tarea para la casa hicieron de manera independiente 13 mapas relacionados con ese tema (Figura 7). 


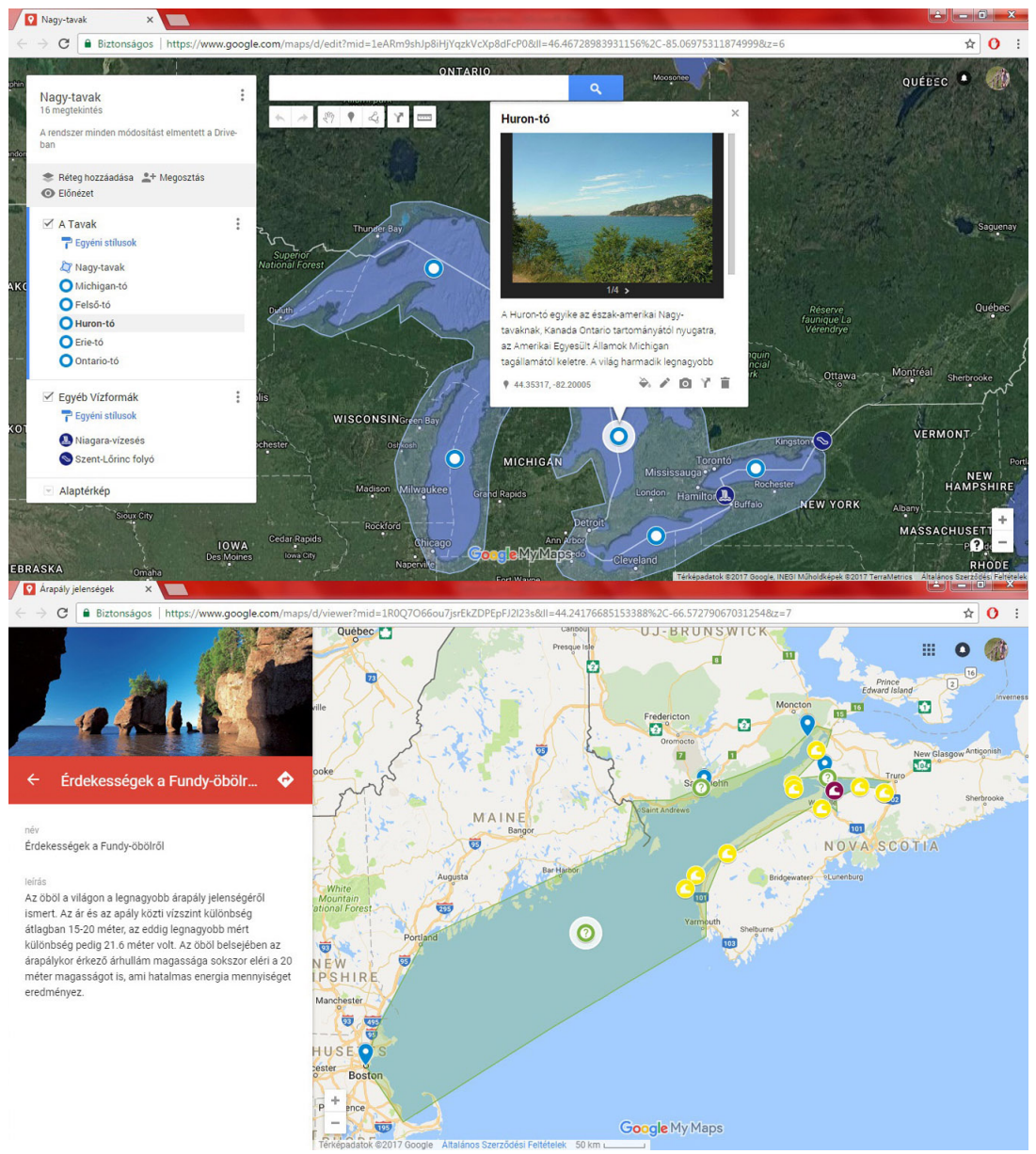

Figura 7. Mapas de temas relacionados con la Geografia del Agua hechos en Google Maps por alumnos húngaros (Reyes y Kiss, 2018).

Cuando hablamos de servicios de mapas en la web, tampoco se puede olvidar un segmento de los Sistemas de Información Geográfica que comienza a cobrar también popularidad: los llamados GIS Online, cuyo representante más notorio es la página ArcGIS Online (https://www.arcgis.com/index.html). Esta página web ofrece la posibilidad a cualquier persona de registrarse y usar de manera gratuita una versión muy simplificada del sistema ArcGIS, diseñada para su uso en la nube web y por cualquier persona que desee crear un mapa sencillo basándose en datos temáticos. ArcGIS Online también tiene un llamado "Atlas Vivo del Mundo" (Living Atlas of the World) que nos permite el acceso gratuito a numerosos mapas de diferentes temas 
que pueden ser utilizados en la educación. ArcGIS Online incluye también una opción que tal vez sea la más conocida: la creación de story maps, que son materiales multimedia interactivos que se realizar usando diferentes diseños. Utilizando estos story maps se puede incluso crear un atlas escolar en línea, como quedó demostrado con uno de los proyectos de investigación desarrollado por universidades españolas en los últimos años: “Atlas Digital Escolar: aprender Geografía en Educación Secundaria con ArcGIS Online" (Figura 8) cuya idea general se accede en la siguiente dirección: http://atlasescolar.maps.arcgis.com/apps/MapJournal/index.html?appid= 77ae3efc94174a2fb216abda32b564f4 (González et al., 2016).

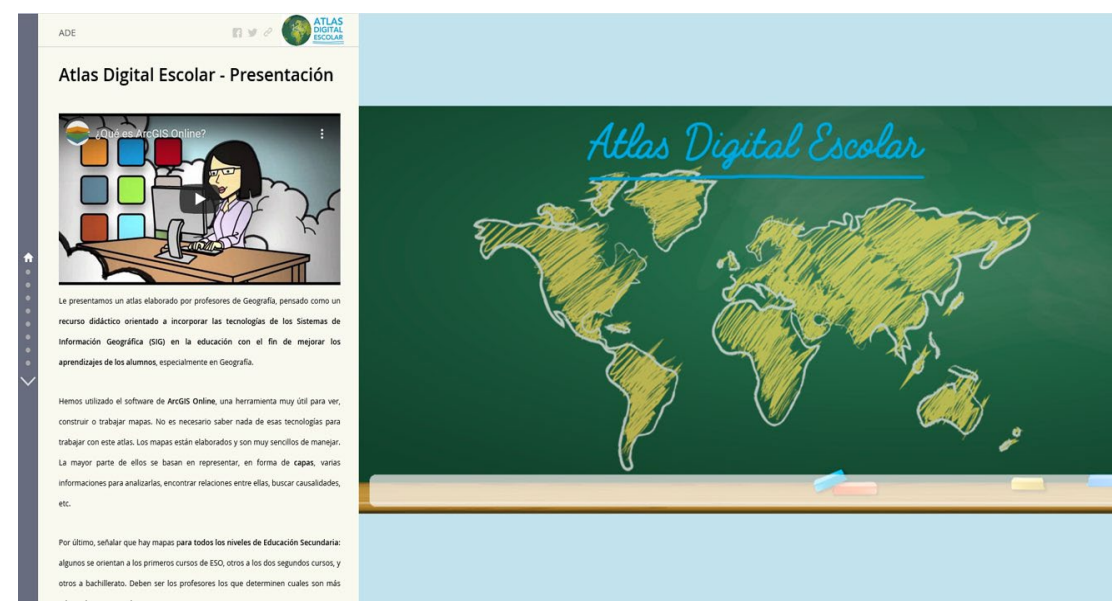

Figura 8. Portada del Atlas Digital Escolar.

\section{A manera de epílogo}

Las investigaciones no se limitan a las opciones anteriormente descritas y ya se buscan nuevos horizontes para experimentar soluciones aún más novedosas. Yendo más allá de la web, se comienza a estudiar cómo aplicar las tecnologías más modernas en la cartografía, abriendo el camino para su implementación en la educación. En el 2019 tuve la oportunidad de escuchar las ideas expuestas por un joven investigador búlgaro, Nikolai Yonov, sobre las posibilidades que la realidad aumentada (Augmented Reality, AR) nos ofrece para complementar la visualización de un atlas escolar con informaciones temáticas (datos, imágenes, modelos tridimensionales, videos, sonidos, etc.) recogidas y actualizadas a tiempo real (Yonov, 2019). ¿Podrá aplicarse esta tecnología de manera masiva en un futuro cercano? Debemos confiar en que se pueda dar una respuesta positiva a esta interrogante en los próximos años. 


\section{Bibliografía}

Anderson, J., Carrière, J. y LeSann, J. (2003). "A Pilot Electronic School Atlas of Quebec, Canada", International Research in Geographical and Environmental Education, 12(4): 383-390. https://doi.org/10.1080/10382040308667552

Bandrova, T. (2006). Atlas de Geografia y Economía para 11 y 12 grados, Sofía. Datamap.

Barros de Sousa, I. (2014). "Geotecnologias e recursos de multimídia no ensino de cartografia: percepção socioambiental do Rio Alcântara no Município de São Gonçalo/RJ", tesis de grado, Universidade do Estado do Rio de Janeiro.

Gede, M., Ungvári, Zs. and Zentai, L. (2013). Virtual Globes Museum 2.0 - Adding the Power of Community, Proceedings of the 26th International Cartographic Conference, 600 pp., Dresden.

Miguel González, R., Lázaro, M.L., Gil, J., Buzo, I. y Moreno, C. (2016). Atlas Digital Escolar: aprender Geografia con ArcGIS Online. https://doi.org/10.14198/GeoAlicante2015.68

Kocsis, K., Márton, M. y Jordan, P. (2019). "Geographic/Thematic Content”, in The Atlas Cookbook. Recuperado de https://atlas.icaci.org/awards-and-publications/atlas-cookbook/

Koop, O. (1993). "Electronic Atlas Production Tools", in Klinghammer, I., Zentai, L. y Ormeling, F. (eds.), Proceedings of the Joint ICA Seminar on Electronic Atlases, pp. 129-137.

Kraak, M.-J. and Ormeling, F. (1996). Cartography: visualization of spatial data, Essex, Longman.

Kriz, K. (2008). "Cartographic Design Issues utilizing Google Earth for Spatial Communication", en Proceedings of the 6th ICA Mountain Cartography Workshop, Lenk, Switzerland, pp. 129-131.

Lastória, A.C. (2007). "A cartografia escolar e a concepção de atlas escolar municipal”, en Dialogus, 3: 111-126. Recuperado de http://sites.ffclrp.usp.br/laife/ atlas.htm

Martinelli, M. (2008). Um atlas geográfico escolar para o ensino-aprendizagem da realidade natural e social. Portal da Cartografia, 1(1): 21-34. Recuperado de http://www.uel.br/revistas/uel/index.php/portalcartografia

Mândruţ, O. (2018). Atlas Geografic Şcolar, Bucarest, Corint.

Ormeling, F. (1996). "Functionality of electronic school atlases", Seminar on Electronic Atlases II. Recuperado de http:/lazarus.elte.hu/hun/doktoran/ferjan/1997electronic.doc

(2014). "Atlases", in Ormeling, F. y Rystedt, B. (eds), The World of Maps. Recuperado de https://icaci.org/publications/the-world-of-maps/the-world-ofmaps-english/ 
Oxford University Press (2019). Oxford Student Atlas for India, Third Edition, Oxford.

Patton, J.C. (1999). "The American School Atlas: 1784-1900", Cartographic perspectives, 33(2): 4-32.

Raisz, E. (1948). General Cartograph, New York, McGraw-Hill.

- (1962). Principles of Cartography, New York, McGraw-Hill.

Reichenbacher, T. (2011). "The World in your pocket - towards a Mobile Cartography”, in Proceedings of ICC 2011. Recuperado de http://icaci.org/files/documents/ICC_proceedings/ICC2001/icc2001/file/f16005.pdf.

Reyes, J; Kiss, J. (2018). "Ideas y experiencias sobre el uso de servicios de mapas web en la educación”, en Boletim Paulista de Geografia (99): 86-101, São Paulo.

Reyes Nuñez, J.J. (2013). "Smartphone-based school atlas?”, Cartographica, 48(2): 126-133. University of Toronto Press.

Safiza, S.B.K.B. (2007). "Cartographic representation on small devices”, RGI 149 Report no. 2, Delf University of Technology.

Tichelaar, T. y Ormeling, F. (2009). Geographical names and education: school world atlases. Recuperado de http://www.stagn.de/static/UNGEGN/2009/11 Geographical\%20names\%20and\%20education.pdf

Voženilek, V. (2014). "Aspects of the Thematic Atlas Compilation”, in Brus, J., Vondrakova, A. y Voženilek, V. (eds.), Modern Trends in Cartography: Selected Papers of CARTOCON 2014, Heidelberg. Editorial Springer, pp. 3-12.

Wiegand, P. (2006). "Learning and teaching with maps", New York, Routledge.

Yonov, N. (2019). "School Atlas with Augmented Reality", Proc. Int. Cartogr. Assoc., 2: 150. https://doi.org/10.5194/ica-proc-2-150-2019 
